\title{
Evaluación probabilística de licuación en arenas de la ciudad de Piura en Perú
}

\author{
Probabilistic evaluation of sands liquefaction in Piura city in Peru
}

Fecha de entrega: 27 de febrero 2017

Fecha de aceptación: 30 de agosto 2017

\section{Denisse Dianet Campos-Muñoz ${ }^{1}$, Alfonso Mariano Ramos-Cañón ${ }^{1}$ y Luis Felipe Prada-Sarmiento ${ }^{2}$}

\footnotetext{
${ }^{1}$ Instituto Geofísico, Pontificia Universidad Javeriana, Carrera 7 No. 42 - 27, piso 7, Edificio Lorenzo Uribe, Bogotá, Colombia, denisse_campos@javeriana.edu.co,a-ramos@javeriana.edu.co

${ }^{2}$ Departamento de Ingeniería Civil, Pontificia Universidad Javeriana, Carrera 7 No. 40 - 62, Edificio 42, Bogotá, Colombia, 1f.pradas@javeriana.edu.co
}

El método de Seed et al. (1985) es uno de los métodos utilizados para el cálculo de licuación y depende de la relación de esfuerzos cíclicos resistentes CRR y actuantes CSR para obtener el margen de seguridad. Con mucha frecuencia, esta medida de seguridad se supone como determinística y no considera la incertidumbre de las solicitaciones y de las propiedades resistentes del suelo. Los trabajos que se han desarrollado desde una perspectiva probabilística suponen funciones de densidad de probabilidad para CRR y CSR, y coeficientes de variación para las propiedades del suelo reportados en la literatura de zonas diferentes a las del caso de aplicación. Este trabajo presenta un procedimiento aplicado a la ciudad de Piura en Perú que incluye la incertidumbre de todas las variables aleatorias involucradas en el cálculo de licuación obtenidos de datos de la zona de estudio, ofreciendo un análisis de incertidumbre completo. El análisis probabilístico utiliza los métodos de FORM y simulaciones de Montecarlo. La probabilidad de licuación máxima es de 9.5\% y se obtiene que el valor de $N$ del SPT y nivel freático son las variables que más influyen en la probabilidad de licuación por su alta incertidumbre y la forma funcional en el margen de seguridad.

Palabras clave: probabilidad de licuación, series de Taylor, simulaciones de Monte Carlo
A method to estimate liquefaction is the one proposed by Seed et al. (1985). This method depends on the cyclic resistance ratio $C R R$ and the cyclic shear stress ratio CSR to calculate the safety margin. Frequently, this measure of safety is assumed deterministic and it is not contemplated the uncertainty of the seismic loads and soils resistance properties. When the probabilistic approach is implemented, often, probability density distribution functions for CRR, CSR and coefficients of variation of the soils properties are assumed by some authors. This paper presents a procedure applied to the city of Piura in Peru that includes the uncertainty of all random variables for liquefaction calculations. The information obtained from the area of study provides sufficient data to develop a complete analysis of uncertainty. In order to perform the probabilistic analysis, it was used the FORM and Monte Carlo methods. The maximum liquefaction probability is $9.5 \%$ and the random variables with more influence in the result for high uncertainty and functional form in the safety margin are $N$ of the SPT and ground water level. Keywords: liquefaction probability, Taylor's series, Monte Carlo simulations

\section{Introducción}

Piura se encuentra ubicada en la zona conocida como el cinturón de fuego y es una de las ciudades más atacadas por el Fenómeno El Niño (ver Figura 1). La extensión de la zona de estudio es de aproximadamente
$25 \mathrm{~km}^{2}$, el nivel freático es alto y sube con la ocurrencia de este fenómeno provocando inundaciones. El suelo se caracteriza por poseer arenas pobremente gradadas SP y arenas limosas SM. Estas condiciones facilitan la ocurrencia de procesos de licuación ante 
la materialización de la amenaza sísmica (Hurtado, 2011). Las evaluaciones de posibilidad de licuación que se han realizado en Piura (Ascencio, 2012; Pinto, 1998) consideran la situación más crítica sin tomar en cuenta la convolución de distintos escenarios alimentados por la variabilidad de los parámetros actuantes y resistentes en el suelo. Además, NASEM (2016) menciona explícitamente la necesidad de tener en cuenta la incertidumbre en los análisis de licuación que se realizan con base en la resistencia del suelo.

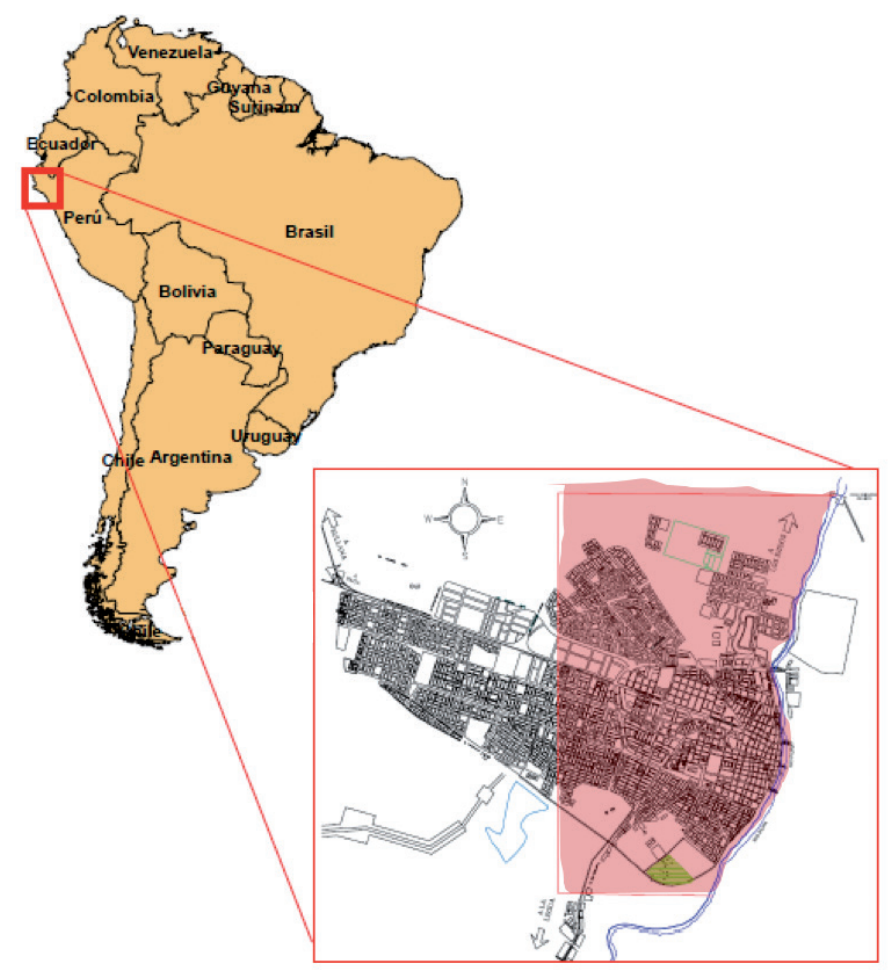

Figura 1: Ubicación de la ciudad de Piura y zona de estudio achurada

En muchos países latinoamericanos, la medida de seguridad de la posibilidad de licuación (factor de seguridad o margen de seguridad) utilizada en el diseño considera un valor determinístico sin realizar un análisis específico de la variabilidad de las fuerzas actuantes (solicitación) y de las propiedades mecánicas del suelo (resistencia). La norma MVCS (2006) recomienda la metodología determinística de Seed et al. (1985) para la obtención del factor de seguridad. La norma NSR (2010) recomienda el uso de métodos determinísticos o probabilísticos reconocidos internacionalmente para el cálculo de licuación. Por otro lado, existen códigos como el
Eurocode (2005), EHE (1998) y ROM (2005) que ya tienen incorporado el diseño basado en confiabilidad de la parte estructural. La metodología propuesta en este documento hace un acercamiento desde el cálculo de licuación de suelos como aplicación práctica.

El método de cálculo de licuación propuesto por Seed et al. (1985) ha sido ampliamente usado (e.g. Robertson y Wride, 1998; Andrus y Stokoe II, 2000; Idriss y Boulanger, 2006) porque propone funciones que dependen de $N$ del SPT, ensayo utilizado en distintos países latinoamericanos como Perú, Ecuador, Colombia, Brasil y Chile (Yanez et al., 2015; Menezes et al., 2006). Las ecuaciones y gráficos propuestos en la metodología de Seed et al. (1985) parten de una serie de eventos de licuación y no licuación ocurridos en Japón, China y América como producto de eventos sísmicos. Dada la naturaleza empírica del procedimiento propuesto por Seed et al. (1985) se observa un nivel de incertidumbre inherente en su aplicación. Un ejemplo es su aplicación en el sismo de Loma Prieta ocurrido en 1989 que causó problemas de licuación en la bahía de Monterrey en California. Los resultados arrojaron que las zonas licuables no se podían distinguir de las no licuables a través de una línea claramente demarcada, sino que es una franja y que el factor de seguridad para un análisis de licuación no es un único valor, sino que presenta variabilidad. Al realizar el análisis probabilístico en esta zona se concluyó que para un factor de seguridad de 1.5, la probabilidad de licuación es de 1\% (Juang et al., 1999). Jha y Suzuki (2008) hacen una recopilación de los métodos más usados para calcular la probabilidad de licuación y los aplican a zonas de edificios de estudio de televisión de Nepal. Ellos usan coeficientes de variación de la aceleración y magnitud del sismo, $N$ del SPT y densidad seca obtenidos de la literatura técnica. Los autores establecen que el resultado de la probabilidad de licuación por los métodos de series de Taylor, estimación puntual y simulación de Montecarlo presenta una mayor dependencia del CRR que del CSR debido a la mayor variabilidad de los parámetros del suelo que las características del sismo. Sin embargo, se sabe que la variabilidad de los parámetros actuantes o resistentes en el cálculo de licuación depende de la 
zona de estudio y el uso de coeficientes de variación de la literatura no son necesariamente representativos de la zona de análisis. De la misma manera, Boulanger e Idriss (2012) desarrollan un análisis probabilístico y suponen que la relación de esfuerzos cíclicos actuantes y resistentes son funciones de distribución lognormal y normal, respectivamente. La desviación estándar de las funciones se obtiene a partir de coeficientes de variación de sólo dos variables que son la aceleración de sismo y el $N$ del SPT reportados en la literatura. Dicho trabajo concluye que la caracterización de la amenaza sísmica y de los parámetros del suelo son componentes muy importantes para cualquier análisis probabilístico de amenaza por licuación debido a que la incertidumbre epistémica es más baja que la incertidumbre de las variables sísmicas y de caracterización del sitio. Gutiérrez et al. (2003) realizan un análisis de confiabilidad al usar la metodología de Seed y estudian la variabilidad de los parámetros geotécnicos que intervienen en el cálculo de la relación $C R R$ basado en coeficientes de variación estimados de datos de presas de United States Bureau of Reclamation dams. En este trabajo utilizan el método de series de Taylor simplificado para calcular la probabilidad de licuación considerando que la relación CSR es constante en la zona de estudio.

Estudios posteriores prefieren ajustar los eventos de licuación y no licuación registrados en Japón de distinta manera utilizando herramientas estadísticas tales como regresiones logísticas y mapeos bayesianos y relacionar la probabilidad de licuación con valores de $N$ del SPT, $q_{c}$ del CPT y velocidades de corte $V_{s}$. Al graficar los resultados por ambos métodos, se recomienda usar el mapeo bayesiano, basado en el teorema de Bayes, porque hereda las características del método determinístico y permite la fácil obtención del factor de seguridad a partir de la probabilidad de licuación y viceversa (Juang et al., 2002). Estas herramientas no consideran la incertidumbre de las variables por separado, sólo obtienen la variabilidad del factor de seguridad que es calculado de manera determinística. Asimismo, Hwang et al. (2004) obtuvieron funciones de densidad de probabilidad de tipo lognormal para la relación $C S R$ y $C R R$ a partir de una ley de atenuación empírica en una zona de Taiwan y datos de eventos de licuación y no licuación generados por el sismo de Chi - Chi. Se utilizó el método de series de Taylor para estimar la probabilidad de licuación del margen de seguridad, que presenta una distribución de probabilidad de tipo lognormal. La metodología para obtener la función de probabilidad del $C R R$ sólo considera los eventos de licuación y no licuación de la zona y no desarrolla la incertidumbre de las variables del suelo que participan en los procesos de licuación.

El presente trabajo propone un procedimiento para la caracterización del margen de seguridad en el cálculo de licuación en arenas, como una función probabilística obtenida del estudio estadístico de las variables involucradas y propias de la zona de estudio sin considerar la profundidad de análisis debido a que su influencia en el coeficiente de reducción de esfuerzo $r_{\mathrm{d}}$ es mayor a profundidades superiores a $10 \mathrm{~m}$ (Gutiérrez et al., 2003), y el presente estudio no llega a evaluar hasta esa profundidad. Estas variables son los valores del número de golpes $N$ del ensayo SPT, la densidad, profundidad del nivel freático, el porcentaje de finos del suelo eólico de Piura, la aceleración máxima inducida por sismo y magnitud del sismo. Esta propuesta se diferencia de lo reportado porque no supone funciones de distribución de probabilidad para el CSR (Boulanger e Idriss, 2012) ni coeficientes de variación para obtener el CRR (Jha y Suzuki, 2008; Juang et al., 1999), sino que involucra la variabilidad de los esfuerzos cíclicos resistentes y actuantes propios de la zona, sin considerar la variabilidad epistémica de la función de desempeño que ya ha sido estudiada por Hwang et al. (2004) y Boulanger e Idriss (2012). Para el análisis probabilístico, se utilizan los métodos de series de Taylor y simulaciones de Montecarlo. Se determina la probabilidad de licuación por metro de profundidad para diferentes perforaciones y se identifica la variable que tiene mayor influencia en la incertidumbre del cálculo de probabilidad de licuación en la ciudad de Piura. La probabilidad de licuación obtenida en cada exploración se interpola usando la técnica de distancia inversa ponderada IDW y se visualiza en el mapa de la ciudad de Piura. 


\section{Determinación del potencial de licuación}

El cálculo del potencial de licuación se realiza utilizando la metodología de Seed et al. (1985) y se define el margen de seguridad $M S$ con:

$M S=C R R-C S R$

donde $C R R$ es la relación de resistencia cíclica que presenta el suelo y $C S R$ es la relación de esfuerzos cíclicos impuestos por un sismo. La evaluación consiste en analizar las relaciones de esfuerzos cíclicos que resisten los suelos donde se supone ocurrirá el sismo y se comparan con la relación de esfuerzos cíclicos que generan los sismos. La relación CSR se define como el esfuerzo cortante máximo generado por el sismo entre el esfuerzo efectivo vertical de una columna de suelo.

$C S R=\frac{\tau}{\sigma_{v}^{\prime}}=0.65 \frac{\sigma_{v}}{\sigma_{v}^{\prime}} \frac{a_{\max }}{g} \frac{r_{d}}{M S F}$

donde $\sigma_{v}$ es el esfuerzo vertical total del suelo y $\sigma_{v}^{\prime}$ es el esfuerzo vertical efectivo del suelo, $a_{\max }$ es la aceleración horizontal máxima en la superficie del terreno generada por un sismo, g es la aceleración de la gravedad y $r_{\mathrm{d}}=1-0.01 z$ es un factor de corrección de esfuerzo por profundidad, el cual decrece de 1 hasta 0.9 para $10 \mathrm{~m}$ de profundidad (Seed e Idriss, 1971), donde $z$ es la profundidad de análisis en metros. Youd et al. (2001) recomienda que el factor de escala $M S F$ se calcule en función de la magnitud del sismo $M$ como:

$$
M S F=\frac{10^{2.24}}{M^{2.56}}
$$

La relación $C R R$ expresa la capacidad del suelo para resistir licuación. Esta relación está en función del número de golpes $N$ del SPT y para valores de $N$ menores a 30 golpes/pie, satisface:

$$
C R R=\frac{1}{34-N_{160 c s}}+\frac{N_{160 c s}}{135}+\frac{50}{\left[10 N_{160 c s}+45\right]^{2}}-\frac{1}{200}
$$

El grado de drenaje durante la ejecución del ensayo de penetración SPT, disminuye con el incremento del contenido de finos, por lo que el $N$ medido subestima la resistencia a la licuación en arenas limosas. Para compensar este efecto se recomienda aumentar el $N$ del SPT a medida que aumenta el contenido de finos del suelo granular, es decir, el valor de $N_{160 c s}$ considera un factor de corrección por contenido de finos dado por:

$$
N_{160 c s}=e^{\left(1.76-\frac{190}{F C^{2}}\right)}+\left(0.99+\frac{F C^{2}}{1000}\right) N_{160}
$$

donde $F C$ es el contenido de finos y $N_{160}=$ $N C_{\mathrm{N}} C_{\mathrm{E}} C_{\mathrm{R}} C_{\mathrm{S}} C_{\mathrm{B}}$ corresponde al número de golpes/pie normalizado a una presión de confinamiento de 100 $\mathrm{kPa}$ aproximadamente y una energía de martillo de $60 \%$, donde $C_{\mathrm{E}} C_{\mathrm{R}} C_{\mathrm{S}} C_{\mathrm{B}}$ son los factores de corrección por energía del martillo, longitud de varillaje, método de muestreo y diámetro de la perforación, los cuales se consideran constantes (Youd et al., 2001). $C_{\mathrm{N}}=$ $\left(p_{\mathrm{a}} / \sigma_{\mathrm{v}}\right)^{0.5}$ es el factor de corrección por presión de confinamiento en suelos granulares (Liao y Whitman, 1986). Al incluir todas las variables aleatorias en (1) se obtiene la expresión que corresponde al margen de seguridad en función de todas las variables aleatorias.

$$
\begin{aligned}
& M S=\frac{1}{34-N_{160 c s}}+\frac{N_{160 c s}}{135}+\frac{50}{\left[10 N_{160 c s}+45\right]^{2}}- \\
& \frac{1}{200}-0.65 \frac{\rho z}{\rho z-\rho_{w} z_{w}} \frac{a_{\max }}{g} \frac{r_{d}}{M S F}
\end{aligned}
$$

\section{Caracterización de la incertidumbre de las variables aleatorias del Margen de Seguridad de licuación MS}

Las variables aleatorias del margen de seguridad $M S$ en (6) son la densidad seca del suelo $\rho$, profundidad del nivel freático $z_{w}$, aceleración máxima en la superficie $a_{\text {max }}$, magnitud del sismo $M$, valor de $N$ del SPT y contenido de finos $F C$. Las razones por las que se considera variables aleatorias son: a) $\rho$ a una misma profundidad presenta variabilidad espacial, se evidencia en los datos utilizados en el presente trabajo y en la literatura (Jones et al., 2002), b) $z_{\mathrm{w}}$ depende directamente de los niveles de precipitación de la ciudad que a su vez genera la infiltración de agua, c) $a_{\text {max }}$ en la superficie depende de la magnitud del sismo, la 
distancia epicentral y el hipocentro, las cuales presentan variabilidad para cada evento sísmico y además existe una probabilidad de ocurrencia para estos eventos, d) $M$ es diferente para cada evento sísmico y además existe una probabilidad de ocurrencia para estos eventos, e) $N$ posee incertidumbre y se evidencia en los datos utilizados en el presente trabajo y en la literatura (Baecher y Christian, 2003) y $F C$ porque la granulometría de las arenas a una misma profundidad es distinta y se evidencia en los datos utilizados en el presente trabajo y en el trabajo de Gutiérrez et al. (2003).

$M S$ tiene 6 variables aleatorias cuya incertidumbre debe ser caracterizada por medio de sus momentos estadísticos y de sus funciones de densidad de probabilidad. Cada variable se ajustó a funciones de densidad de probabilidad utilizando la prueba Kolmogorov - Smirnov, considerando un nivel de confiabilidad de $95 \%$.

En las áreas costeras, por estar más cerca de la zona de Benioff, los focos suelen ser superficiales y conforme se avanza hacia el continente pasan a ser intermedios y profundos. Los epicentros de los temblores ocurridos en la costa piurana en las últimas décadas se han localizado entre $5 \mathrm{~km}$ y $183 \mathrm{~km}$; y los hipocentros han estado a menos de $120 \mathrm{~km}$ de profundidad, la mayoría de los sismos ocurridos han sido superficiales. Existe evidencia de licuación de suelos en la ciudad de Piura por los sismos de 1857, 1912 y 1970, que generó grietas con aberturas de hasta $30 \mathrm{~cm}$ y saltos de $25 \mathrm{~cm}$ y la formación de volcanes de arena entre 0.6 y $1 \mathrm{~m}$ de diámetro (Silgado, 1978; Hurtado, 2011).

Los datos para caracterizar $M$ y $a_{\text {max }}$ correspondientes al departamento de Piura, se obtuvieron del Instituto Geofísico del Perú IGP y USGS en el período de 1970 - 2015. La magnitud del sismo utilizada en el análisis es magnitud momento $M_{\mathrm{w}}$ que varía de 4 a 6.5 y la aceleración varía de 0.01 a $0.44 \mathrm{~g}$, ambas variables se ajustaron a funciones de distribución beta y los parámetros de las funciones se muestran en la Tabla 1. $M$ y $a_{\max }$ se asumen como variables independientes, lo cual permite obtener una probabilidad de licuación mayor. La función beta obtenida para la aceleración sísmica es compatible con las curvas de probabilidad de excedencia típicas obtenidas en evaluaciones de amenaza sísmica (Gamarra, 2009). La función de distribución beta es muy versátil porque los datos se pueden adecuar con sólo variar los parámetros $q$ y $r$ que son una medida de la asimetría y kurtosis de las funciones generadas, y se acota con los parámetros $a$ y $b$ que corresponden al límite inferior y superior, respectivamente. La función beta esta definida por las siguientes expresiones:

$$
\begin{aligned}
& f_{X}(x)=\frac{1}{B(q, r)} \frac{(x-a)^{q-1}(b-x)^{r-1}}{(b-a)^{q+r-1}} \\
& B(q, r)=\int_{0}^{1} x^{q-1}(1-x)^{r-1} d x
\end{aligned}
$$

Tabla 1: Parámetros de la función y estadísticos para $M$ y $a$

\begin{tabular}{|c|c|c|}
\hline Parámetros de la función & $M$ & $a_{\max }, \mathrm{g}$ \\
\hline$q$ & 0.70 & 0.80 \\
\hline$r$ & 3.80 & 11.50 \\
\hline$b$ & 6.50 & 0.45 \\
\hline$a$ & 4.30 & 0.00 \\
\hline Estadísticos & \multicolumn{2}{|l}{} \\
\hline Promedio & 4.64 & 0.03 \\
\hline Desviación estándar & 0.34 & 0.03 \\
\hline Coeficiente de variación & 0.07 & 1.04 \\
\hline
\end{tabular}

Para determinar los momentos estadísticos de $z_{\mathrm{w}}$, se considera que el nivel encontrado en cada una de las perforaciones es el valor esperado y se supone que el coeficiente de variación del nivel freático es igual al coeficiente de variación de la precipitacion diaria anual en la ciudad suponiendo que el aumento del nivel de agua sólo depende de la infiltración de agua de lluvia (SGC, 2015). Esta suposición se realiza con base en la característica desértica de la zona y en los depósitos de arena que van desde muy sueltos a medios con valores de $N$ menores a 20 golpes/ pie hasta los $4 \mathrm{~m}$ de profundidad, que permite la infiltración rápida de agua. Esta afirmación se basa en los resultados de Puga (2012) que encontró a través de ensayos de permeámetro de carga constante que arenas con densidad relativa menor presentan mayores permeabilidades.

La variabilidad de la precipitación anual se caracterizó 
con el registro de períodos lluviosos de 45 años (1971 - 2015) en la estación del Radar Universidad de Piura, ubicada en el nor occidente de la ciudad. En la Figura 2 se presenta la precipitación anual en la estación del Radar de la Universidad de Piura y se observa que los años 1983 y 1998 fueron los de mayor precipitación anual. Estos son los años en los que se presentó el fenómeno El Niño y que produjo inundaciones y la caída de puentes en la zona del río Piura. Al analizar todos los datos de precipitación anual se obtiene un coeficiente de variación de $259 \%$, por lo que se separaron los datos de los años de fenómeno El Niño de los datos de años sin este fenómeno y se obtuvó las funciones de probabilidad que se ajustan a ambos escenarios por separado.

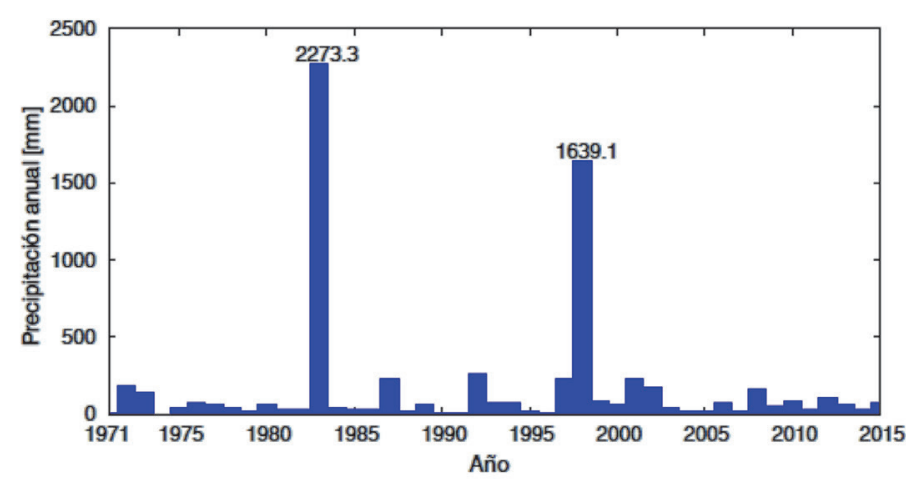

Figura 2: Precipitación anual registrado por la estación Radar UdeP en los años 1971 a 2015.

Los datos de precipitación anual de años sin fenómeno El Niño se ajustaron a una distribución lognormal y los datos de años con fenómeno El Niño se ajustaron a una distribución normal, ambos ajustes cumplen con un nivel de confiabilidad de $95 \%$ de acuerdo a la prueba K-S. En la Tabla 2 se presentan los parámetros de la función y estadísticos de los datos.

La distribución de probabilidad lognormal es estrictamente positiva y con el aumento de la desviación estándar aumenta la asimetría. La función está dada por la siguiente expresión:

$f_{X}=\frac{1}{\xi x \sqrt{2 \pi}} \exp \left[-\frac{1}{2}\left(\frac{\ln x-\lambda}{\xi}\right)^{2}\right]$

donde los parámetros $\lambda$ y $\xi$ están en función de la media y desviación estándar de todos los datos.
Tabla 2: Parámetros de la función y estadísticos para la precipitación anual

\begin{tabular}{|c|c|c|}
\hline $\begin{array}{l}\text { Parámetros de la } \\
\text { función }\end{array}$ & $\begin{array}{l}\text { Precipitación años } \\
\text { con fenómeno El } \\
\text { Niño, mm }\end{array}$ & $\begin{array}{l}\text { Precipitación años } \\
\text { sin fenómeno El } \\
\text { Niño, mm }\end{array}$ \\
\hline $\begin{array}{l}\text { Distribución } \\
\text { lognormal }\end{array}$ & & $\mathrm{x}$ \\
\hline $\begin{array}{l}\text { Distribución } \\
\text { normal }\end{array}$ & $\mathrm{x}$ & \\
\hline$\lambda$ & & 3.927 \\
\hline$\xi$ & & 0.815 \\
\hline \multicolumn{3}{|l|}{ Estadísticos } \\
\hline Promedio & 154.54 & 70.74 \\
\hline $\begin{array}{l}\text { Desviación } \\
\text { estándar }\end{array}$ & 399.95 & 68.74 \\
\hline $\begin{array}{l}\text { Coeficiente de } \\
\text { variación }\end{array}$ & 2.59 & 0.972 \\
\hline
\end{tabular}

$$
\lambda=\ln \mu_{\mathrm{x}}-0.5 \xi^{2} \quad \text { y } \xi=\sqrt{\ln \left(1+C O V^{2}\right)}
$$

donde $C O V$ es el coeficiente de variación. La desviación estándar de la variable $z_{\mathrm{w}}$ se obtiene a partir del coeficiente de variación de la precipitación anual para el escenario sin fenómeno El Niño, porque es el que tiene mayor frecuencia de ocurrencia en la ventana de tiempo analizada en la ciudad de Piura (45 años). Para el método de simulaciones de Montecarlo se utilizó una distribución de probabilidad lognormal con $\lambda$ de acuerdo a (10) para cada perforación y $\xi$ de 0.815 . Para caracterizar los momentos estadísticos y determinar la función de distribución de probabilidad de la variable aleatoria $N$ del ensayo SPT, se recopilaron datos de 64 exploraciones SPT que ocupan un área de $25 \mathrm{~km}^{2}$ de la ciudad de Piura, realizadas por el Laboratorio de ensayos de materiales de la Universidad de Piura entre los años 1983 y 2010 y cuyo perfil estratigráfico presenta arenas pobremente gradadas SP y arenas limosas SM en 4 m o más de profundidad. Los datos de $N$ se definieron por profundidad y corresponden a un tipo de suelo obtenido con un mismo equipo. En la Tabla 3 de presentan los datos de $N$ ajustados a funciones de densidad de probabilidad lognormal y beta. Se observa que a partir de $5 \mathrm{~m}$ de profundidad, los valores de $N$ de SPT son mayores a 30 golpes/pie y de acuerdo a la metodología de Seed et al. (1985), la ecuación de $C R R$ no aplica porque estos suelos no presentan problemas de licuación. 
Tabla 3: Parámetros de la función y estadísticos para el valor de $N$ del SPT por profundidad

\begin{tabular}{|c|c|c|c|c|c|c|c|c|}
\hline $\begin{array}{l}\text { Función de } \\
\text { distribución de } \\
\text { probabilidad }\end{array}$ & $1 \mathrm{~m}$ & $2 \mathrm{~m}$ & $3 \mathrm{~m}$ & $4 \mathrm{~m}$ & $5 \mathrm{~m}$ & $6 \mathrm{~m}$ & $7 \mathrm{~m}$ & $8 \mathrm{~m}$ \\
\hline $\begin{array}{l}\text { Distribución } \\
\text { lognormal }\end{array}$ & $\mathrm{x}$ & $\mathrm{x}$ & $\mathrm{x}$ & $\mathrm{x}$ & $\mathrm{x}$ & $\mathrm{x}$ & $\mathrm{x}$ & \\
\hline $\begin{array}{l}\text { Distribución } \\
\text { beta }\end{array}$ & & & & & & & & $\mathrm{x}$ \\
\hline \multicolumn{9}{|l|}{$\begin{array}{l}\text { Parámetros de } \\
\text { la función }\end{array}$} \\
\hline$q$ & & & & & & & & 3 \\
\hline$r$ & & & & & & & & 4 \\
\hline$b$ & & & & & & & & 100 \\
\hline$a$ & & & & & & & & 16 \\
\hline$\lambda$ & 2.28 & 2.58 & 2.84 & 3.11 & 3.41 & 3.73 & 3.87 & \\
\hline$\xi$ & 0.24 & 0.26 & 0.22 & 0.21 & 0.18 & 0.15 & 0.16 & \\
\hline \multicolumn{9}{|l|}{ Estadísticos } \\
\hline Promedio & 11 & 15 & 19 & 25 & 33 & 45 & 52 & 66 \\
\hline $\begin{array}{l}\text { Desviación } \\
\text { estándar }\end{array}$ & 5.77 & 8.09 & 9.36 & 12.2 & 14.7 & 18 & 21.6 & 22.1 \\
\hline $\begin{array}{l}\text { Coeficiente } \\
\text { de variación }\end{array}$ & 0.52 & 0.54 & 0.49 & 0.49 & 0.45 & 0.40 & 0.41 & 0.33 \\
\hline
\end{tabular}

Los coeficientes de variación de $N$ son mayores en las capas superficiales y van disminuyendo con la profundidad. Harr (1984) reportan $C O V$ de $N$ entre 40 y $45 \%$ y Kulhawy y Trautman (1996) reportan valores entre 14 y 100\% considerando la variabilidad del equipo, procedimiento y variabilidad natural. Los valores encontrados en la ciudad de Piura se encuentran dentro de lo reportado en la literatura y se asocia principalmente a la incertidumbre del equipo y procedimiento dado que corresponde a un solo tipo de suelo.

Para caracterizar los momentos estadísticos y la función de densidad de probabilidad de la variable aleatoria densidad seca $\rho$, se recopilaron datos de ensayos de densidad de las muestras de las exploraciones tipo SPT obtenidas a cada metro de profundidad. Estos valores se ajustaron a funciones de probabilidad lognormal y beta con los parámetros indicados en la Tabla 4.

Gutiérrez et al. (2003) reportan COV de 6\% para la densidad seca de materiales SP - SW y entre 5 y $10 \%$ para la densidad seca de materiales SM - ML. La densidad seca de los materiales SP y SM de la ciudad de Piura presenta incertidumbres reflejada en $\mathrm{COV}$ menores al 6\%. Para la variable aleatoria de contenido de finos $F C$, se recopilaron datos de ensayos de granulometría de 43 muestras de las exploraciones SPT obtenidas a cada metro de profundidad. Los datos se ajustaron a funciones de probabilidad lognormal con los parámetros indicados en la Tabla 5.

Tabla 4: Parámetros de la función y estadísticos para la densidad por profundidad

\begin{tabular}{|c|c|c|c|c|c|c|c|c|}
\hline $\begin{array}{l}\text { Función de } \\
\text { distrib. de } \\
\text { probabilidad }\end{array}$ & $1 \mathrm{~m}$ & $2 \mathrm{~m}$ & $3 \mathrm{~m}$ & $4 \mathrm{~m}$ & $5 \mathrm{~m}$ & $6 \mathrm{~m}$ & $7 \mathrm{~m}$ & $8 \mathrm{~m}$ \\
\hline $\begin{array}{l}\begin{array}{l}\text { Distribución } \\
\text { lognormal }\end{array} \\
\end{array}$ & & $\mathrm{x}$ & $\mathrm{x}$ & $\mathrm{x}$ & $\mathrm{x}$ & $\mathrm{x}$ & $\mathrm{x}$ & $\mathrm{x}$ \\
\hline $\begin{array}{l}\text { Distribución } \\
\text { beta }\end{array}$ & $\mathrm{x}$ & & & & & & & \\
\hline \multicolumn{9}{|l|}{$\begin{array}{l}\text { Parámetros } \\
\text { de la } \\
\text { función }\end{array}$} \\
\hline$q$ & 2 & & & & & & & \\
\hline$r$ & 3.2 & & & & & & & \\
\hline$b$ & 1.8 & & & & & & & \\
\hline$a$ & 1.55 & & & & & & & \\
\hline$\lambda$ & & 0.56 & 0.65 & 0.66 & 0.68 & 0.70 & 0.70 & 0.72 \\
\hline$\xi$ & & 0.002 & 0.001 & 0.001 & 0.001 & 0.001 & 0.001 & 0.0001 \\
\hline \multicolumn{9}{|l|}{ Estadísticos } \\
\hline Promedio & 1.65 & 1.75 & 1.91 & 1.94 & 1.97 & 2.01 & 2.02 & 2.05 \\
\hline \begin{tabular}{|l|}
$\begin{array}{l}\text { Desviación } \\
\text { estándar }\end{array}$ \\
\end{tabular} & 0.04 & 0.08 & 0.04 & 0.05 & 0.06 & 0.05 & 0.04 & 0.01 \\
\hline \begin{tabular}{|l|}
$\begin{array}{l}\text { Coeficiente } \\
\text { de variación }\end{array}$ \\
\end{tabular} & 0.03 & 0.05 & 0.02 & 0.03 & 0.03 & 0.03 & 0.02 & 0.01 \\
\hline
\end{tabular}

Tabla 5. Parámetros de la función y estadísticos para el contenido de finos por profundidad.

\begin{tabular}{|l|c|c|c|c|c|c|c|c|}
\hline $\begin{array}{l}\text { Parámetros de } \\
\text { la función }\end{array}$ & $1 \mathrm{~m}$ & $2 \mathrm{~m}$ & $3 \mathrm{~m}$ & $4 \mathrm{~m}$ & $5 \mathrm{~m}$ & $6 \mathrm{~m}$ & $7 \mathrm{~m}$ & $8 \mathrm{~m}$ \\
\hline$\lambda$ & 1.11 & 1.16 & 1.19 & 1.18 & 1.19 & 1.02 & 1.04 & 1.25 \\
\hline$\xi$ & 0.31 & 0.29 & 0.26 & 0.22 & 0.23 & 0.16 & 0.21 & 0.29 \\
\hline \begin{tabular}{l} 
Estadísticos \\
\hline Promedio
\end{tabular} & 3.55 & 3.67 & 3.73 & 3.65 & 3.70 & 2.99 & 3.14 & 4.03 \\
\hline $\begin{array}{l}\text { Desviación } \\
\text { estándar }\end{array}$ & 2.13 & 2.11 & 2.01 & 1.82 & 1.89 & 1.23 & 1.53 & 2.35 \\
\hline $\begin{array}{l}\text { Coeficiente de } \\
\text { variación }\end{array}$ & 0.60 & 0.57 & 0.54 & 0.50 & 0.51 & 0.41 & 0.49 & 0.58 \\
\hline
\end{tabular}

El coeficiente de variación para el contenido de finos $F C$ es de $60 \%$, variabilidad que es mayor a la reportada por Gutiérrez et al. (2002) que presentan COV de hasta $31.5 \%$. Esto se debe a que las arenas de Piura son de origen aluvial y eólico, lo anterior genera que los depósitos aluviales tengan lentes de limos 
muy delgados mientras que los depósitos eólicos no suelen presentar finos. Dicha disparidad en el origen de formación de los depósitos se refleja en la mayor variabilidad del contenido de finos.

\section{Métodos para propagación de incertidum- bre}

Los métodos para análisis de confiabilidad permiten la obtención del índice de confiabilidad y probabilidad de falla del margen de seguridad definido con (6), la cual indica la probabilidad de licuación en la zona de estudio. Los métodos usados en este trabajo son primer orden segundo momento o también conocido como series de Taylor y simulaciones de Montecarlo.

\section{Método de primer orden segundo momento - Series de Taylor}

Este método describe la media, varianza y desviación estándar de una función como los primeros términos de una expansión de la serie de Taylor. Se supone que $\left(x_{\mathrm{i}}-\mu x_{\mathrm{i}}\right)$ de cada variable aleatoria son pequeños $\left(x_{\mathrm{i}}\right.$ es la variable aleatoria y $\mu x_{i}$ es la esperanza de la variable aleatoria), por lo que los cuadrados y potencias más altas también lo son y pueden ignorarse. La esperanza del margen de seguridad $E[M S]$ se obtiene evaluando el valor medio de cada variable aleatoria en (6).

$$
E[M S] \approx M S\left(\mu a_{\max }, \mu M, \mu \rho, \mu z_{w}, \mu N, \mu F C\right)
$$

La varianza del margen de seguridad $\operatorname{Var}[M S]$ se obtiene con la derivada parcial de la función de desempeño con respecto a la variable aleatoria, suponiendo que las variables son independientes entre sí.

$$
\begin{aligned}
& \operatorname{Var}[M S]=\sigma_{M S}^{2} \\
& \sigma_{M S}^{2} \approx \sigma_{a_{\max }^{2}} \frac{\partial M S^{2}}{\partial a_{\max }}+\sigma_{M}^{2} \frac{\partial M S^{2}}{\partial M}+\sigma_{\rho}^{2} \frac{\partial M S^{2}}{\partial \rho}+ \\
& \sigma_{z_{w}}^{2} \frac{\partial M S^{2}}{\partial z_{w}}+\sigma_{N}^{2} \frac{\partial M S^{2}}{\partial N}+\left.\sigma_{F C}^{2} \frac{\partial M S^{2}}{\partial F C}\right|_{\mu a_{\max }, \mu M, \mu \rho, \mu z_{w}, \mu N, \mu F C}
\end{aligned}
$$

La ecuación (12) presenta la multiplicación de la varianza de cada variable aleatoria y el cuadrado de la derivada parcial, evaluada en los valores medios de las variables. El número de derivadas parciales del margen de seguridad corresponden a la cantidad de variables aleatorias.

\section{Simulaciones de Monte Carlo}

El método de Monte Carlo permite determinar la distribución probabilística de la función de desempeño mediante la generación de números aleatorios basado en las variables aleatorias de la función. Este método plantea conocer la probabilidad de ocurrencia de un evento, realizando el experimento un número suficiente de veces, determinando la función de desempeño como una distribución probabilística de los resultados obtenidos en los experimentos realizados. Conociendo la distribución de probabilidad de las variables aleatorias de la función de desempeño, se muestrean aleatoriamente valores de estas variables aleatorias, y se evalúa determinísticamente la función. El proceso previamente explicado se repite un número de veces suficientemente grande para conseguir la convergencia de la distribución de probabilidad de la función de desempeño (Hidalgo y Pacheco, 2011; Arevalo et al., 2014; Prada et al., 2011). Esta convergencia se evalúa en el primer y segundo momento estadístico para obtener la cantidad de simulaciones requeridas para que la variación de estos sea mínima.

La aplicación del Método Monte Carlo es eficiente cuando se cuenta con las herramientas computacionales para efectuar grandes volúmenes de cálculos numéricos, debido a que se consigue una mejor aproximación de la función de densidad de probabilidad resultante cuando se incrementan el número de simulaciones realizadas. Se obtuvo convergencia de los momentos estadísticos del margen de seguridad para 100000 simulaciones como se observa en la Figura 3.

\section{Probabilidad de licuación}

El cálculo de probabilidad de licuación se realizó por el método de series de Taylor y simulaciones de Monte Carlo en las 64 perforaciones de la ciudad de Piura. Esta probabilidad de licuación es anual debido a que la incertidumbre de $a_{\max }, M$ y precipitación se realizó por año en una ventana temporal desde 1970 a 2015. 


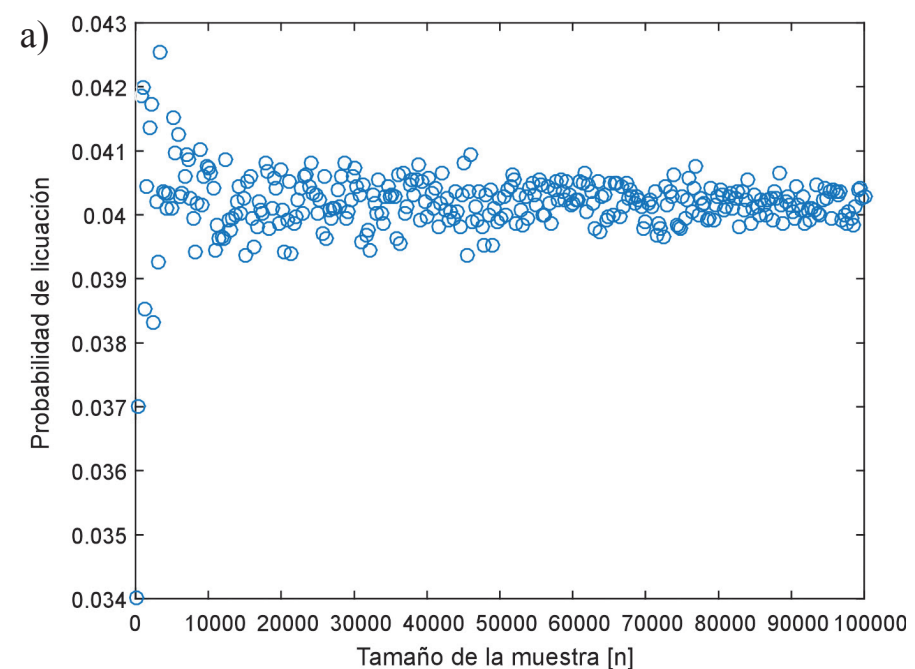

b)

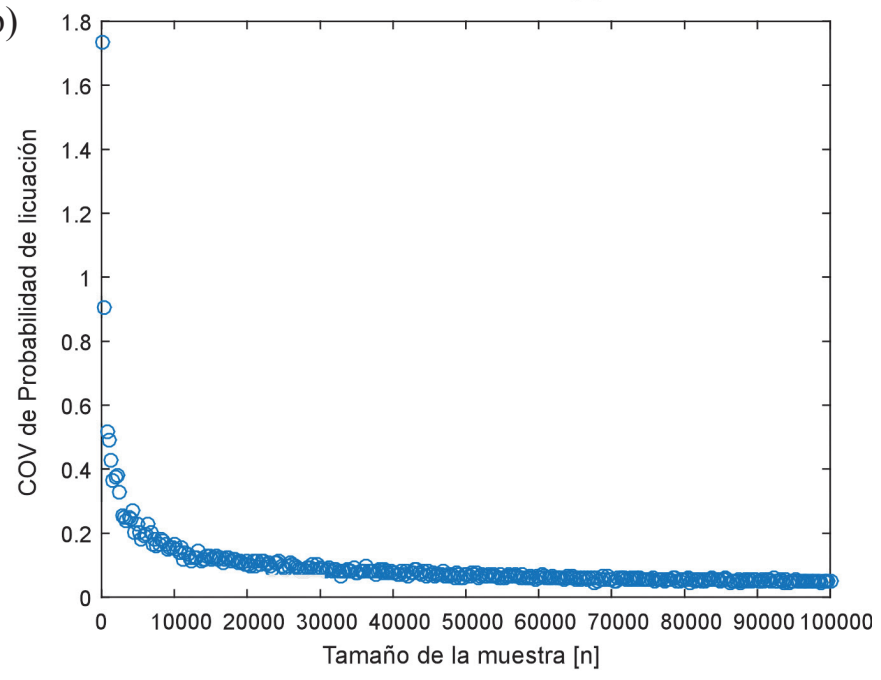

Figura 3: Número de simulaciones de Monte Carlo para a) la probabilidad de licuación y b) su coeficiente de variación

A manera de ejemplo se presenta el procedimiento para una perforación ubicada en las coordenadas 9428008.00 latitud sur y 540100 longitud este. La ecuación para obtener $C R R$ es útil para valores de $N$ menores a 30 golpes/pie, y de acuerdo a los estadísticos de $N$ en la zona se puede aplicar (4) hasta $4 \mathrm{~m}$ de profundidad, por lo que se obtiene la probabilidad de licuación hasta esa profundidad. El método de series de Taylor requiere del cálculo de las derivadas parciales de $M S$ con respecto a cada variable aleatoria usando (12). La incertidumbre dada por la función de desempeño se puede cuantificar con el valor de estas derivadas y las variables de mayor influencia en la incertidumbre de la función de desempeño siguen el siguiente orden: $a_{\max }, \rho_{\mathrm{d}}, z_{\mathrm{w}}$ y $N$. Para determinar la variable más influyente en la desviación estándar del margen de seguridad se analiza cada término de la sumatoria presentada en (12) y se obtiene su relación con respecto a la varianza del $M S$. Los resultados se presentan en la Tabla 6 e indica que las variables más influyentes son $N \mathrm{y} z_{\mathrm{w}}$, este último es el más influyente en un $94 \%$ a $1 \mathrm{~m}$ de profundidad y a $4 \mathrm{~m}$ de profundidad ambas variables son igualmente influyentes con un $50 \%$ para $z_{\text {w }}$ y $49 \%$ para el $N$.

Tabla 6: Porcentaje de influencia de las variables aleatorias en la incertidumbre de $M S$

\begin{tabular}{|c|c|c|c|c|c|c|}
\hline $\begin{array}{c}\text { Prof., } \\
\mathrm{m}\end{array}$ & $a_{\max }, \%$ & $M, \%$ & $z_{\mathrm{w}}, \%$ & $\rho, \%$ & $N, \%$ & $F C, \%$ \\
\hline 1 & 0.032 & 0.002 & 94.19 & 0.016 & 5.76 & 0.000 \\
\hline 2 & 0.008 & 0.000 & 86.47 & 0.165 & 13.36 & 0.000 \\
\hline 3 & 0.006 & 0.000 & 54.88 & 0.059 & 46.05 & 0.003 \\
\hline 4 & 0.021 & 0.001 & 50.83 & 0.015 & 49.00 & 0.002 \\
\hline
\end{tabular}

Para conocer la influencia de los componentes $C S R$ y $C R R$ en $M S$, se obtiene la varianza de cada componente y se analiza con respecto a la suma de varianzas y los resultados indican que el $C R R$ presenta mayor incertidumbre en la probabilidad de licuación que el CSR (ver Figura 4). Este resultado se debe a que las variables que intervienen en el cálculo del $C R R$ son $z_{\text {w }}, N, \rho$ y $F C$, que incluye las dos variables más influyentes; mientras que para el cálculo de CSR interviene $z_{\mathrm{w}}, a_{\max }, M \mathrm{y} \rho$, que tiene sólo una variable influyente. El CSR posee una influencia de 3.3\% a $1 \mathrm{~m}$ de profundidad y disminuye hasta $0.01 \%$ a $4 \mathrm{~m}$ de profundidad, por lo que la incertidumbre del $C R R$ es la más influyente en el cálculo de licuación de la ciudad de Piura.

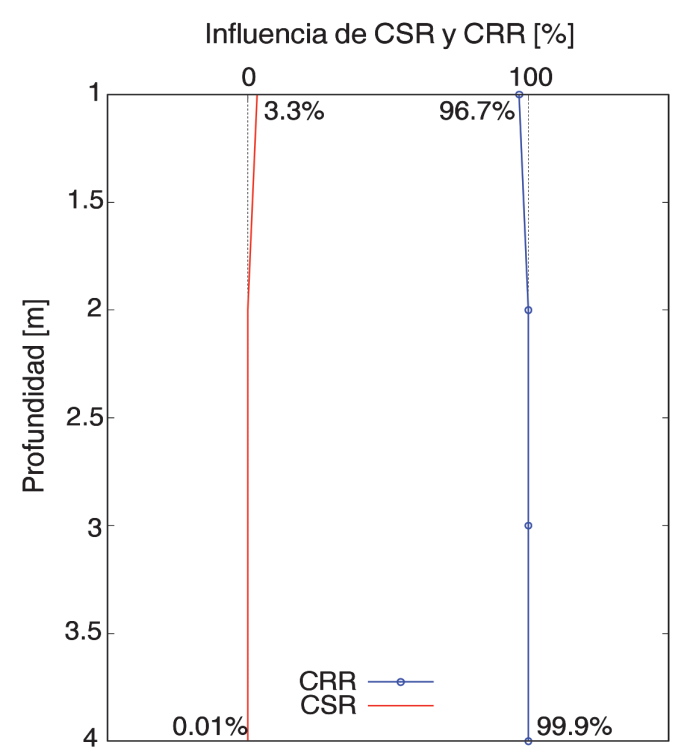

Figura 4: Variación de la influencia de la incertidumbre del $C R R$ y CSR en $M S$ de la licuación en profundidad 
Jha y Suzuki (2008) encontraron que la incertidumbre del $C R R$ es mayor al CSR en ciudades como Nepal, Singhdurbar y Kathmandu debido a que el coeficiente de variación de $N$, tomado de la literatura para diferentes tipos de suelos, es mayor a los coeficientes de $M$ y $a_{\text {max }}$ del sismo. La variabilidad del $C R R$ encontrada en la ciudad de Piura se debe en gran medida a la incertidumbre de $z_{\mathrm{w}}$, asociada a la precipitación en la zona, y al $N$ que está relacionado con el suelo, equipo y procedimiento del ensayo SPT. El análisis estadístico de las variables del sismo y del suelo en la ciudad de Piura permite que el cálculo de la probabilidad de licuación integre la incertidumbre propia de la zona de todas las variables actuantes y resistentes que intervienen en el margen de seguridad, sin requerir los coeficientes de variación reportados en la literatura. Suponiendo que $M S$ de licuación de suelos en la ciudad de Piura se caracteriza por una distribución lognormal, se obtiene el índice de confiabilidad y la probabilidad de licuación hasta $4 \mathrm{~m}$ de profundidad en la perforación. Para las simulaciones de Monte Carlo, se realizó un código de programación para la generación de números aleatorios de las distintas distribuciones a las que se aproximan las 6 variables aleatorias de la función de desempeño. Se realizaron 100000 simulaciones de Monte Carlo a cada profundidad, número a partir del cual la probabilidad de licuación y coeficiente de variación del margen de seguridad convergen.

Al comparar los resultados por el método de series de Taylor y simulación de Monte Carlo se observa que la probabilidad de licuación disminuye con la profundidad. La Figura 5 presenta la integración de la incertidumbre de las variables que generan procesos de licuación en la ciudad de Piura hasta $4 \mathrm{~m}$ de profundidad. Se puede observar que al suponer que el margen de seguridad tiene una distribución lognormal en el método de series de Taylor, el histograma de frecuencias del margen de seguridad y la probabilidad de licuación es parecida a la obtenida por el método de simulaciones de Monte Carlo. La máxima diferencia presentada es de $3 \%$ a $1 \mathrm{~m}$ y $4 \mathrm{~m}$ de profundidad debido a que la función lognormal y el histograma de frecuencias obtenido por Monte Carlo poseen momentos estadísticos de cuarto orden diferentes. (a)
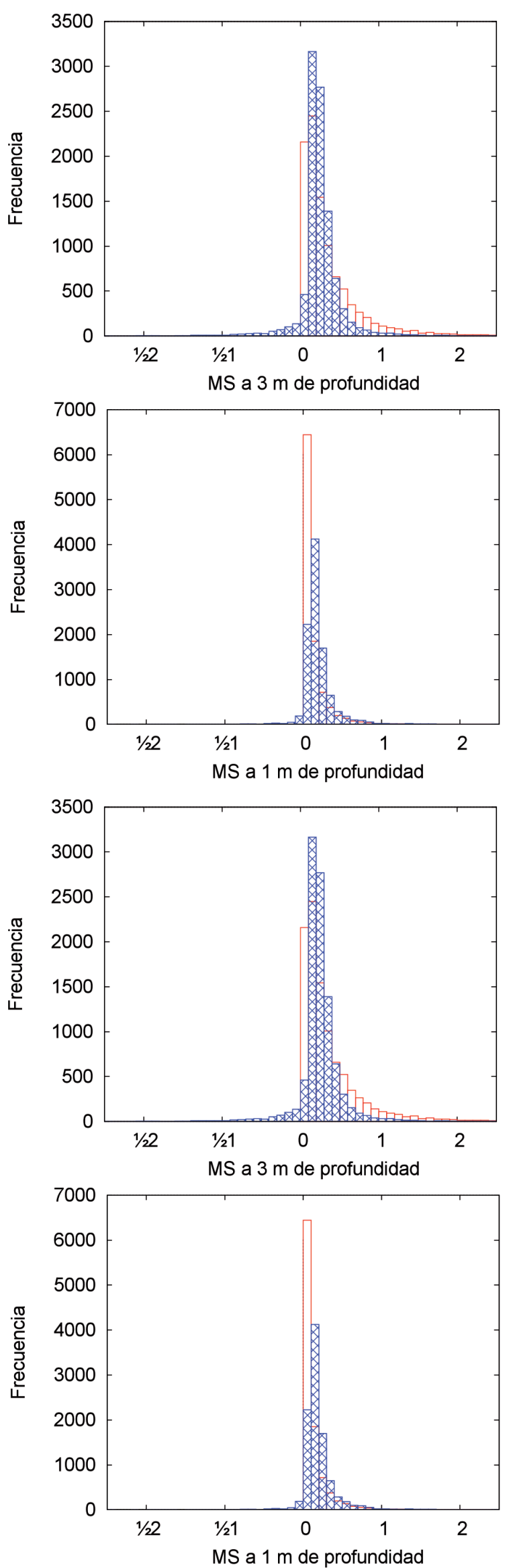
(b)

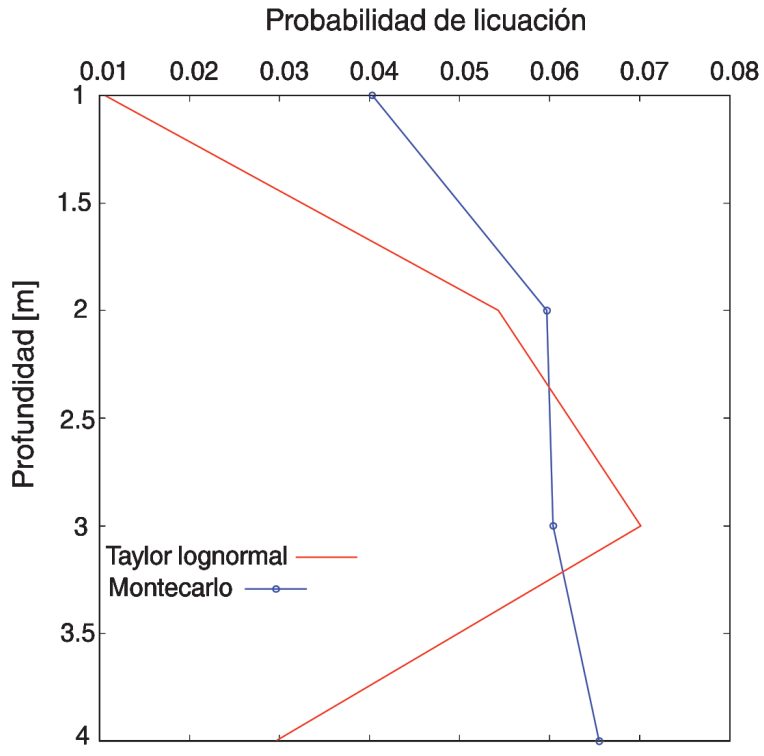

Figura 5: a) Histogramas de frecuencias del MS y b) probabilidad de licuación por profundidad

A $1 \mathrm{~m}$ de profundidad la curtosis es mayor por el método de series de Taylor y a $4 \mathrm{~m}$ de profundidad la curtosis es mayor por el método de simulaciones de Monte Carlo. Si se compara la diferencia de probabilidad de licuación obtenida con la diferencia encontrada en los resultados del trabajo de Jha y Suzuki (2008), se puede indicar que es pequeña y se debe a la forma de las funciones de probabilidad del margen de seguridad obtenidas a distintas profundidades.

Para visualizar la probabilidad de licuación en la ciudad de Piura se realizó una interpolación usando la técnica de distancia inversa ponderada IDW. En la Figura 6 se presenta el mapa de la ciudad de Piura con los contornos de probabilidad de licuación obtenido por simulaciones de Monte Carlo.

De manera general, se observa que la probabilidad de licuación máxima es de $9.5 \%$, disminuye con la profundidad y en ciertas zonas se mantiene o aumenta a $4 \mathrm{~m}$ de profundidad debido a que la incertidumbre de $N$ es mayor a esa profundidad registrándose valores de $N$ desde 5 hasta 60 golpes/pie (arenas sueltas y arenas densas). A manera de ejemplo, se ha utilizado los datos de la ciudad de Piura en la metodología propuesta por Gutiérrez et al. (2003), la cual utiliza el método de series de Taylor simplificado y supone una distribución lognormal del MS. Las expresiones que utilizaron los autores para obtener el CRR y $C S R$

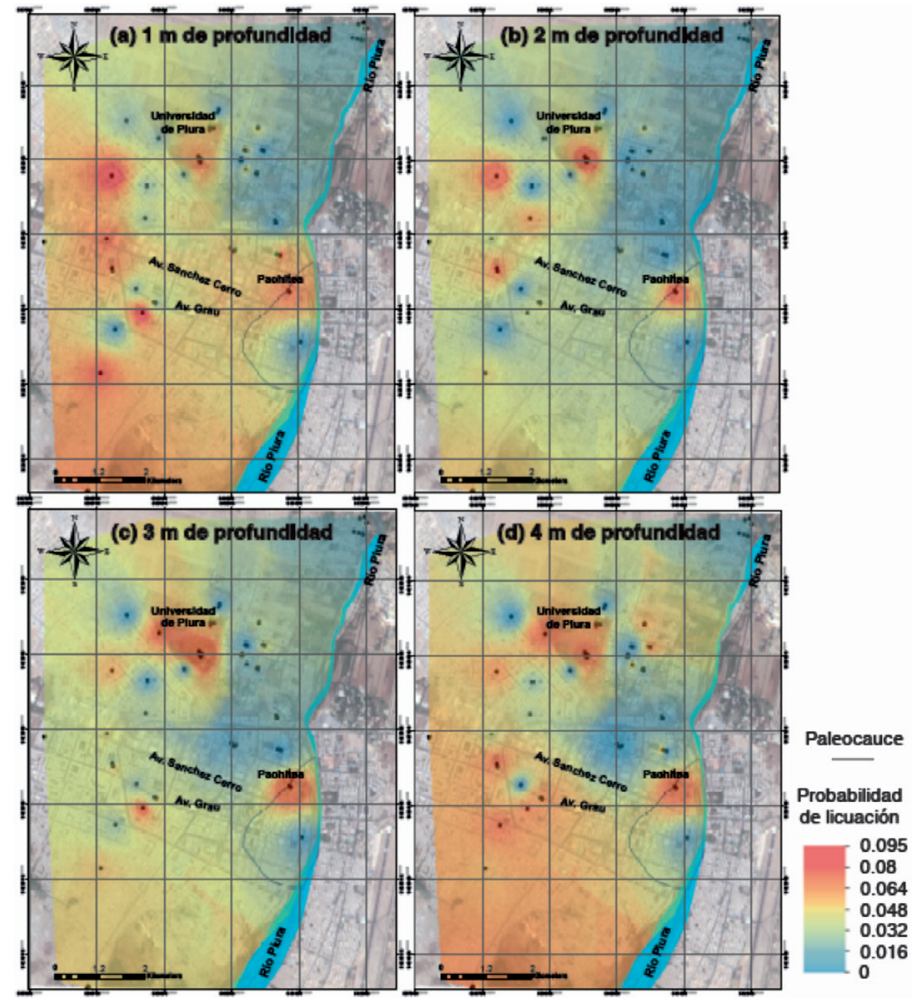

Figura 6: Probabilidad de licuación en la ciudad de Piura para distintas profundidades

son iguales a (2) y (4) y el ejemplo de aplicación considera al CSR como determinista y con un valor de 0.1. La probabilidad de licuación máxima usando los datos de Piura en el método de series de Taylor simplificado resulta ser $10.4 \%$, valor cercano a $9.5 \%$ obtenido en este trabajo por simulaciones de Monte Carlo. Dado que el ejemplo de Gutiérrez et al. (2003) considera el CSR como determinista, se espera que el resultado sea parecido al obtenido por simulaciones de Monte Carlo en Piura, en el que se observó que el $C R R$ presenta mayor influencia en la probabilidad de licuación que el $C S R$.

De acuerdo a la geología local, el cuaternario reciente de Piura está constituido por depósitos aluviales extendidos por las márgenes, terrazas y zonas aledañas al río Piura y depósitos eólicos de mayor extensión lejos del curso actual del río (Campos, 2011). Los depósitos aluviales están compuestos de limos arenosos y arena pobremente gradada con lentes de limos. En Piura existen dos grandes cursos aluviales de recorrido aproximadamente paralelo. Uno de ellos lo constituye el actual cauce del río Piura y el otro es un antiguo lecho del río (paleocauce - Figura 6) 
que se identifica superficialmente por constituir una franja topográficamente baja (30 msnm) que atraviesa la urbanización Santa Isabel, Pachitea, urbanización Grau y 04 de enero y llega finalmente al anteriormente conocido Barrio Sur. Las zonas situadas fuera de estos cursos aluviales tienen predominancia de material eólico y en general están ubicadas en el oeste de Piura. La Figura 6 muestra que la probabilidad de licuación en la zona de Pachitea se mantiene con la profundidad y se debe a que los valores de $N$ son bajos y el perfil estratigráfico presenta arenas sueltas hasta 4 $\mathrm{m}$ de profundidad. Por otro lado, los depósitos eólicos en Piura son irregulares por su espesor variado, compacidad y permeabilidad; están compuestos de arenas pobremente gradadas con un contenido de finos que varía de 0.4 a $9.2 \%$. Dentro de la zona de depósitos se observa que las exploraciones ubicadas en zonas descampadas dentro del campus de la Universidad de Piura y en zonas aún no urbanizadas, la probabilidad de licuación es mayor y se mantiene hasta la profundidad analizada debido a que los vientos en la zona permiten la erosión y depositación de los sedimentos libremente. Este movimiento de arenas es continuo y no permite su compactación, y se refleja en la incertidumbre de $N$ que presenta una variación de $49 \%$ a profundidades de 3 y $4 \mathrm{~m}$.

\section{Conclusiones}

El procedimiento presentado para la evaluación probabilística de licuación para la ciudad de Piura en Perú presenta ventajas con respecto a procedimientos anteriormente propuestos dado que por un lado, se tiene en cuenta la totalidad de variables aleatorias que dominan el proceso y la caracterización de la incertidumbre de dichas variables se realiza con base en información de la zona de estudio. Aspectos que no habían sido contemplados simultáneamente en estudios pasados. Las funciones de distribución de probabilidad obtenidas para las variables son distintas a las típicas funciones reportadas en la literatura (distribución normal y lognormal). Las variables se ajustaron a distribuciones lognormales y betas; esta última es la más usada debido a que se puede adecuar a cualquier registro de datos con sólo variar los parámetros de la función.
La incertidumbre generada por la función de desempeño se mide con las derivadas del margen de seguridad de licuación con respecto a cada variable aleatoria e indica que la aceleración máxima $a_{\max }$, profundidad del nivel freático $z_{\mathrm{w}}$, densidad seca $\rho_{\mathrm{d}}$ y $N$ del SPT son las variables más influyentes. Al aplicar el método de series de Taylor se encuentra que las variables de mayor influencia en la desviación estándar del margen de seguridad $M S$ es $N y z_{\text {w }}$, debido a que presentan mayor magnitud en la combinación de la incertidumbre en la zona de estudio y las derivadas parciales. En superficie (1 y $2 \mathrm{~m}$ de profundidad) la variable $z_{\mathrm{w}}$ es más influyente y conforme se avanza en profundidad, ambas variables son igualmente influyentes (4 $\mathrm{m}$ de profundidad). La incertidumbre de la componente $C R R$ es mayor que la de la componente $C S R$ debido a que hereda la variabilidad de $z_{\mathrm{w}}, N, \rho_{\mathrm{d}}$ y contenido de finos $F C$; es decir, el cálculo del $C R R$ involucra las dos variables más influyentes. Esta característica es propia de Piura y aunque es similar a lo encontrado en otros estudios realizados en Nepal, Singhdurbar y Kathmandu, los autores suponen de antemano coeficientes de variación de la variable $N$ mayores a los de las variables $M$ y $a_{\text {max }}$ del sismo (Jha y Suzuki, 2008). Se puede establecer que la componente CSR del margen de seguridad se puede considerar como determinístico en comparación con CRR para la zona de estudio. El método de series de Taylor y simulaciones de Monte Carlo indican que la probabilidad de licuación anual disminuye con la profundidad; y al suponer que la distribución de probabilidad del $M S$ es lognormal la probabilidad de licuación es parecida a la obtenida por simulaciones de Monte Carlo. Esto se debe a que las funciones de probabilidad de las variables son de tipo lognormal y beta (tipo exponencial).

De manera general, la probabilidad de licuación anual en Piura disminuye con la profundidad quedando ciertas zonas en las que la probabilidad de licuación se mantiene incluso a $4 \mathrm{~m}$ de profundidad debido a la gran variabilidad que presenta la variable $N$ que es uno de los factores más influyentes en el margen de seguridad. Si bien es cierto el valor de $N$ aumenta con la profundidad, su variabilidad es mayor reflejándose 
un grado de compacidad de las arenas desde sueltas hasta densas a $4 \mathrm{~m}$ de profundidad.

Existe una zona conocida como Pachitea que presenta una probabilidad de licuación de $9.5 \%$ y se mantiene hasta $4 \mathrm{~m}$ de profundidad en la que la variabilidad del $N$ está relacionado con la geología de Piura que indica la existencia de un paleocauce paralelo al actual curso del río que cruza por esta zona caracterizada por tener un perfil estratigráfico de arenas sueltas. Las zonas descampadas presentan una probabilidad de licuación mayor que se mantiene hasta la profundidad analizada y se debe al constante movimiento de los sedimentos ocasionado por el viento que no permiten la compactación del material y se refleja en una mayor variabilidad del $N$ en estas zonas.

\section{Referencias}

Andrus, R.D. and Stokoe II, K.H. (2000). Liquefaction resistance of soils from shear-wave velocity. Journal of Geotechnical and Geoenvironmental Engineering 126(11), 1015-1025

Arévalo, G., Ramos-Cañón, A. y Prada, L. (2014). Análisis de confiabilidad en un modelo de descarga de silos de almacenamiento mediante el Método de Elementos Discretos DEM. Obras y Proyectos 15, 21-30

Ascencio Saavedra, F. (2012). Aplicación del SIG como herramienta para la prevención de riesgos geotécnicos en la ciudad de Piura. Tesis de grado, Universidad de Piura

Baecher, G.B. and Christian, J.T. (2003). Reliability and statistics in geotechnical engineering. John Wiley \& Sons

Boulanger, R.W. and Idriss, I.M. (2012). Probabilistic standard penetration test-based liquefaction-triggering procedure. Journal of Geotechnical and Geoenvironmental Engineering 138(10), 1185-1195

Campos Muñoz, D.D. (2011). Estudio de la variabilidad del suelo de Piura a través del SPT para la valoración del FS. Tesis de grado, Universidad de Piura

Eurocode (2005). Design of Steel Structures, Part 1-1; General Rules and Rules for Buildings. European Committee for Standardization, Brussels

EHE (1998). Instrucción de hormigón estructural. Ministerio de Fomento, Madrid
Gamarra Rivera, C.A. (2009). Nuevas fuentes sismogénicas para la evaluación del peligro sísmico y generación de espectros de peligro uniforme en el Perú. Universidad Nacional de Ingeniería, Perú

Gutierrez, M. Duncan, J.M., Woods, C. and Eddy, E. (2003). Development of a simplified reliability-based method for liquefaction evaluation. Report USGS Grant N 02HQGR0058, Virginia Polytechnic Institute and State University

Harr, M.E. (1984). Reliability-based design in civil engineering. Henry Shaw Lecture, Department of Civil Engineering, North Carolina State University, Raleigh

Hidalgo Montoya, C.A. y Pacheco de Assis, A. (2011). Herramientas para análisis por confiabilidad en geotecnia: La teoría. Revista Ingenierías Universidad de Medellín 10(18), 69-78

Hurtado, E.A. (2011). Breve historia del fenómeno de licuación de suelos en el Perú. Centro Peruano Japonés de Investigaciones Sísmicas y Mitigación de Desastres CISMID. Guzlop Editoras, Lima

Hwang, J.H., Yang, C.W. and Juang, D.S. (2004). A practical reliability - based method for assessing soil liquefaction potential. Soil Dynamics and Earthquake Engineering 24(9), 761-770

Idriss, I.M. and Boulanger, R.W. (2006). Semi-empirical procedures for evaluating liquefaction potential during earthquakes. Soil Dynamics and Earthquake Engineering 26(2), 115-130

Jha, S. and Suzuki, K. (2008). Reliability analysis of soil liquefaction based on standard penetration test. Computers and Geotechnics 36(4), 589-596

Jones, A.L., Kramer, S. L. and Arduino, P. (2002). Estimation of uncertainty in geotechnical properties for performanced-based earthquake engineering. California: Pacific Earthquake Engineering Research Center

Juang, C.H., Liang, T. and Andrus, R.D. (2002). Assessing probability-based methods for liquefaction potential evaluation. Journal of Geotechnical and Geoenviromental Engineering 128(7), 580-589

Juang C.H., Rosowsky D.V. and Tang W.H. (1999). Reliability - based method for assessing liquefaction potential of soils. Journal of Geotechnical and Geoenviromental Engineering 125(8), 684-689 
Kulhawy, F.H. and Trautmann, C.H. (1996). Estimation of in-situ test uncertainty. Conference on Uncertainty in the Geologic Environment: from theory to practice, ASCE GSP, Madison, 269-286

Liao, S.S. and Whitman, R.V. (1986). Overburden correction factors for SPT in sand. Journal of Geotechnical Engineering 112(3), 373-377

Menezes, S.M., de Carvalho, D. and da Rocha de Albuquerque, P.J. (2006). Analysis of uplift loads of precastconcrete piles in porous soils. Exacta 4 (1), 191-200

MVCS (2006). Reglamento Nacional de Edificaciones E.050 Suelos y Cimentaciones. Ministerio de Vivienda, Construcción y Saneamiento, Perú

NASEM (2016). State of the art and practice in the assessment of earthquake-induced soil liquefaction and its consequences. The National Academies of Sciences, Engineering, and Medicine. The National Academies Press, Washington, DC

NSR (2010). Normas Colombianas de Diseño y Construcción Sismo Resistente. Asociación Colombiana de Ingeniería Sísmica, Bogotá, Colombia

Pinto Zegarra, C. F. (1998). Identificación de zonas con potencial de licuación de suelos en la ciudad de Piura con ensayos SPT. Tesis de grado, Universidad de Piura

Prada, L., Ramos, A., Solaque, B. y Caicedo, B. (2011). Confiabilidad aplicada al diseño geotécnico de un muro de contención. Obras y Proyectos 9, 49-58

Puga, P.E. (2012). Estudio experimental del coeficiente de permeabilidad en arenas. Proyecto de título, Universidad Católica de la Santísima Concepción, Chile

Robertson, P.K. and Wride, C. E. (1998). Evaluating cyclic liquefaction potential using the cone penetration test. Canadian Geotechnical Journal 35(3), 442-459
ROM (2005). Recomendaciones geotécnicas para obras marítimas y portuarias ROM 0.5-05. Puertos del Estado, España

Seed, H.B. and Idriss, I.M. (1971). Simplified procedure for evaluating soil liquefaction potential. Journal of Soil Mechanics and Foundations Division 97(9), 1249-1273

Seed, H.B., Tokimatsu, K., Harder, L.F. and Chung, R.M. (1985). Influence of SPT procedures in soil liquefaction resistance evaluations. Journal of Geotechnical Engineering 111(12), 1425-1445

SGC (2015). Guía metodológica para estudios de amenaza, vulnerabilidad y riesgo por movimientos en masa. Servicio Geológico Colombiano, Bogotá

Silgado Ferro, E. (1978). Historia de los sismos más notables ocurridos en el Perú (1513-1974). Instituto Geológico Minero y Metalúrgico INGEMMET. Guzlop Editoras, Lima

Yanez, D.G., Massad, F. and Correa, M.R. (2015). Soft soil geotechnical properties in a case study of a large alluvial soft soil improvement in Latin America. 15th Congreso Panamericano de Mecánica de Suelos e Ingeniería Geotécnica, IOS Press, Buenos Aires, 1599-1606

Youd, T.L., Idriss, I.M., Andrus, R.D., Arango, I., Castro, G., Christian, J.T., Dobry, R., Liam Finn, W.D., Harder, L.F., Hynes, M.E., Ishihara, K., Koester, J.P., Liao, S., Marcuson III, W.F., Martin, G.R., Mitchell, J.K., Moriwaki, Y., Power, M.S., Robertson, P.K., Seed, R.B., Stokoe II, K.H. (2001). Liquefaction resistance of soils: summary report from the 1996 NCEER and 1998 NCEER/ NSF workshops on evaluation of liquefaction resistance of soils. Journal of Geotechnical and Geoenvironmental Engineering 127(10), 817-833 